\title{
Their Houses There Without Their Bodies
}

no one is there but someone will be there

transparent houses that have no rooms

or someone was there and is coming back

that have no walls there is nothing but air

or someone is there but can't be seen

or is it water that bubbling that blue

they are not there but they seem to be diving

their clothes are clinging to transparent walls

or swimming in underwater houses

their silvery coppery chairs are balanced

their houses are breathing water their arms

their chairs their jackets a crown are waiting

their bodies cannot be seen they are sitting

and breathing without their bodies they are home 